\title{
ON CONTRAVARIANT FINITENESS OF SUBCATEGORIES OF MODULES OF PROJECTIVE DIMENSION $\leq I$
}

\author{
BANGMING DENG
}

(Communicated by Ken Goodearl)

\begin{abstract}
Let $\wedge$ be an artin algebra. This paper presents a sufficient condition for the subcategory $\mathcal{P}^{i}(\wedge)$ of $\bmod \wedge$ to be contravariantly finite in $\bmod \wedge$, where $\mathcal{P}^{i}(\wedge)$ is the subcategory of $\bmod \wedge$ consisting of $\wedge-$ modules of projective dimension less than or equal to $i$. As an application of this condition it is shown that $\mathcal{P}^{i}(\wedge)$ is contravariantly finite in $\bmod \wedge$ for each $i$ when $\wedge$ is stably equivalent to a hereditary algebra.
\end{abstract}

\section{INTRODUCTION AND PRELIMINARIES}

Throughout this paper, all algebras are artin algebras, all modules are finitely generated left modules, and all subcategories are full subcategories. For an artin algebra $\wedge$, we denote by $\bmod \wedge$ the category of all finitely generated $\wedge$-modules.

The notions of contravariantly and covariantly finite subcategories of $\bmod \wedge$ were first introduced and studied by Auslander and Smal $\phi$ in connection with the study of the existence of almost split sequences in a subcategory of mod $\wedge$ (see [2] and [3]). We now recall these notions from [2].

Definition. A full subcategory $\mathcal{A}$ of $\mathcal{B}$ is said to be

(i) contravariantly finite in $\mathcal{B}$ if for each object $X$ in $\mathcal{B}$, the representable functor $\operatorname{hom}_{\mathcal{B}}(, X)$ restricted to $\mathcal{A}$ is finitely generated as a functor on $\mathcal{A}$,

(ii) covariantly finite in $\mathcal{B}$ if for each object $Y$ in $\mathcal{B}$, the representable functor $\operatorname{hom}_{\mathcal{B}}(Y, \quad)$ restricted to $\mathcal{A}$ is finitely generated, and

(iii) functorially finite if $\mathcal{A}$ is both contravariantly and covariantly finite in $\mathcal{B}$.

For an artin algebra $\wedge$, an interesting class of subcategories of $\bmod \wedge$ is the subcategory $\mathcal{P}^{i}(\wedge)$ which consists of all $\wedge$-modules of projective dimension $\leq i$ for $i \geq 0$, as well as the subcategory $\mathcal{P}^{\infty}(\wedge)$ consisting of all $\wedge$-modules of finite projective dimension. The contravariant and covariant finiteness of $\mathcal{P}^{i}(\wedge)$ and $\mathcal{P}^{\infty}(\wedge)$ is studied by many authors; see, for example, [1], [5], and [6].

The aim of this paper is to present a condition which is sufficient for the subcategory $\mathcal{P}^{i}(\wedge)$ to be contravariantly finite in $\bmod \wedge$. As an application of this condition we show in section 2 that $\mathcal{P}^{i}(\wedge)$ is contravariantly finite in $\bmod \wedge$ for each $i$ when $\wedge$ is stably equivalent to a hereditary algebra. The main idea of the

Received by the editors November 30, 1994.

1991 Mathematics Subject Classification. Primary 16P20, 18 G20.

Supported by the Postdoctoral Science Foundation of China. 
proofs is from [1]. Note that in [1] Auslander and Reiten gave a sufficient condition for $\mathcal{P}^{\infty}(\wedge)$ to be contravariantly finite in $\bmod \wedge$ and proved that $\mathcal{P}^{\infty}(\wedge)$ is contravariantly finite in $\bmod \wedge$ when $\wedge$ is stably equivalent to a hereditary algebra.

The terminology used throughout this article is taken from [1].

\section{A sufficient CONDition for $\mathcal{P}^{i}(\wedge)$ TO Be CONTRAVARIANTLY FInite}

The proofs in this section are analogous to those in sect.4 in [1], but for the completeness of the article, we give here the proofs. Before presenting the sufficient condition, we first give the following more general result.

Proposition 1.1. Suppose that $\underline{a}$ is an ideal in $\wedge$ with $\operatorname{pd}_{\wedge} \wedge / \underline{a} \leq i$ such that if $M$ is a $\wedge$-module with $\operatorname{pd}_{\wedge} M \leq i$, then $M / \underline{a} M$ is a projective $\wedge / \underline{a}-$ module. Let $C$ be $a \wedge / \underline{a}-$ module. Then we have the following.

(a) $A$ map $B \rightarrow C$ in $\bmod \wedge / \underline{a}$ is a right $\mathcal{P}^{0}(\wedge / \underline{a})$-approximation of $C$ if and only if it is a right $\mathcal{P}^{i}(\wedge)$-approximation of $C$.

(b) If $A \rightarrow C$ is a right $\mathcal{P}^{i}(\wedge)$-approximation of $C$, then $A / \underline{a} A \rightarrow C$ is a right $\mathcal{P}^{0}(\wedge / \underline{a})$-approximation of $C$.

Proof. (a) Suppose $f: B \rightarrow C$ is a right $\mathcal{P}^{0}(\wedge / \underline{a})$-approximation of $C$. Since $B$ is a projective $\wedge / \underline{a}$-module and $\operatorname{pd}_{\wedge} \wedge / \underline{a} \leq i$, as a $\wedge$-module $B$ is in $\mathcal{P}^{i}(\wedge)$. Let $g: X \rightarrow C$ be a morphism in $\bmod \wedge$ with $X$ in $\mathcal{P}^{i}(\wedge)$. Then $g$ is the composition of the canonical projection $\pi: X \rightarrow X / \underline{a} X$ and the induced map $g_{1}: X / \underline{a} X \rightarrow C$. Since $X / \underline{a} X$ is a projective $\wedge / \underline{a}$-module, the morphism $g_{1}$ can be lifted to $B$, so $g$ can be lifted to $B$, that is, $f: B \rightarrow C$ is a right $\mathcal{P}^{i}(\wedge)$-approximation of $C$.

Conversely, suppose that a morphism $f: B \rightarrow C$ in $\bmod \wedge / \underline{a}$ is a right $\mathcal{P}^{i}(\wedge)-$ approximation of $C$. Since $\operatorname{pd}_{\wedge} B \leq i$, as a $\wedge / \underline{a}$-module $B=B / \underline{a} B$ is projective. Hence $f: B \rightarrow C$ is a right $\mathcal{P}^{0}(\wedge / \underline{a})-$ approximation of $C$.

(b) This is trivial.

Corollary 1.2. Let a be an ideal in $\wedge$ satisfying the hypothesis of Proposition 1.1. If $\underline{a} S=0$ for each simple $\wedge$-module $S$ with $\operatorname{pd}_{\wedge} S>i$, then $\mathcal{P}^{i}(\wedge)$ is contravariantly finite in $\bmod \wedge$.

Proof. First note that $\mathcal{P}^{i}(\wedge)$ is a resolving subcategory (i.e. $\mathcal{P}^{i}(\wedge)$ satisfying the following three conditions: (a) closed under extension, (b) closed under kernels of surjections, and (c) contains all projective $\wedge$-modules). Then by [1, Proposition 3.7], $\mathcal{P}^{i}(\wedge)$ is contravariantly finite in $\bmod \wedge$ if and only if each simple $\wedge$-module has a right $\mathcal{P}^{i}(\wedge)$-approximation. Suppose $S$ is a simple $\wedge$-module. If $\operatorname{pd}_{\wedge} S \leq i$, we are done. Suppose now $\operatorname{pd}_{\wedge} S>i$; then $S$ is a $\wedge / \underline{a}$-module. Hence there is a right $\mathcal{P}^{0}(\wedge / \underline{a})$-approximation $A \rightarrow S$ of $S$ since $\mathcal{P}^{0}(\wedge / \underline{a})$ is contravariantly finite in $\bmod \wedge / \underline{a}$. By (a) of Proposition 1.1, $A \rightarrow S$ is also a right $\mathcal{P}^{i}(\wedge)$-approximation of $S$. Therefore, $\mathcal{P}^{i}(\wedge)$ is contravariantly finite.

For an artin algebra $\wedge$, we denote by $\Omega(\bmod \wedge)$ the subcategory consisting of the syzygy modules $\Omega(C)$ of all $C$ in $\bmod \wedge$. Further, we denote by $\tau_{\mathcal{A}}(M)$ the trace of a category $\mathcal{A}$ in the module $M$, that is, the submodule of $M$ generated by the images of all maps $A \rightarrow M$ with $A$ in $\mathcal{A}$. Finally, for each $i \geq 0$, we denote by $\underline{a}_{i}$ the trace of $\Omega(\bmod \wedge) \cap \mathcal{P}^{i}(\wedge)$ in $\underline{r}$, i.e. $\underline{a}_{i}=\tau_{\Omega(\bmod \wedge) \cap \mathcal{P}^{i}(\wedge)}(\underline{r})$, where $\underline{r}$ denotes the radical of $\wedge$. It is obvious that $\underline{a}_{i} \subset \underline{r}$ is an ideal in $\wedge$. 
Proposition 1.3. If $\operatorname{pd}_{\wedge} \underline{a}_{i} \leq i$, then $\mathcal{P}^{i+1}(\wedge)$ is contravariantly finite in mod $\wedge$.

Proof. We first observe that $\underline{a}_{i} P=\tau_{\Omega(\bmod \wedge) \cap \mathcal{P}^{i}(\wedge)}(\underline{r} P)$ if $P$ is a projective module, where $\underline{r} P$ is the radical of $P$.

Let $M$ be a $\wedge-$ module with projective dimension $\leq i+1$. We then consider the following exact sequence

$$
0 \longrightarrow \Omega(M) \longrightarrow P \stackrel{f}{\longrightarrow} M \longrightarrow 0
$$

with $f$ a projective cover of $M$. Since $\operatorname{pd}_{\wedge} \Omega(M) \leq i$, one gets that $\Omega(M)$ is in $\Omega(\bmod \wedge) \cap \mathcal{P}^{i}(\wedge)$. Therefore, it holds that $\Omega(M) \subset \tau_{\Omega(\bmod \wedge) \cap \mathcal{P}^{i}(\wedge)}(\underline{r} P)=\underline{a}_{i} P$ and that $P / \underline{a}_{i} P \cong M / \underline{a}_{i} M$, that is, $M / \underline{a}_{i} M$ is a projective $\wedge / \underline{a}_{i}-$ module. The condition $\operatorname{pd}_{\wedge} \underline{a}_{i} \leq i$ implies that $\operatorname{pd}_{\wedge} \wedge / \underline{a}_{i} \leq i+1$. Because each simple $\wedge$-module is annihilated by $\underline{a}_{i}$, one has by Corollary 1.2 that $\mathcal{P}^{i+1}(\wedge)$ is contravariantly finite.

Corollary 1.4. Suppose $\operatorname{pd}_{\wedge} \underline{a}_{i} \leq i$. Then the $\wedge$-modules of projective dimension $\leq i+1$ are the summands of modules $M$ which have filtrations $M=M_{0} \supset M_{1} \supset$ $\cdots \supset M_{n}=0$ such that each subquotient $M_{l} / M_{l+1}$ is an indecomposable projective $\wedge / \underline{a}_{i}-$ module.

Proof. Note that the minimal right $\mathcal{P}^{0}(\wedge / \underline{a})$-approximation of a $\wedge / \underline{a}$-module is its projective cover. It then follows that the $\wedge / \underline{a}-$ projective covers of the simple $\wedge^{-}$ modules are just the minimal right $\mathcal{P}^{i}(\wedge)$-approximations of the simple $\wedge$-modules. The corollary then follows directly from [1, Proposition 3.7].

Combining the above results with the results in [1] and [3], we get the following corollary.

Corollary 1.5. Suppose $\underline{a}_{0}$ is projective. Then $\mathcal{P}^{1}(\wedge)$ has almost split sequences.

Proof. From [1] one knows that $\mathcal{P}^{1}(\wedge)$ is covariantly finite in $\bmod \wedge$ for all artin algebras. By Proposition 1.3, one has that $\mathcal{P}^{1}(\wedge)$ is also contravariantly finite in $\bmod \wedge$, so $\mathcal{P}^{1}(\wedge)$ is functorially finite. Then from [3, Theorem 2.4] it follows that $\mathcal{P}^{1}(\wedge)$ has almost split sequences.

As seen the example in [5], one knows that $\mathcal{P}^{i}(\wedge)$ is not always contravariantly finite. In the following we construct an algebra with finite global dimension $n \geq 2$ which satisfies that $\mathcal{P}^{i}(\wedge)$ are not contravariantly finite for $1 \leq i \leq n-1$.

Example. Let $k$ be an algebraically closed field. For each $n \geq 2$, let $\wedge_{n}$ be given as the path algebra of the following quiver

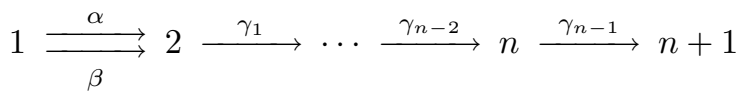

modulo the ideal generated by the paths $\gamma_{1} \alpha$ and $\gamma_{i+1} \gamma_{i}, 1 \leq i \leq n-2$. It is easy to calculate that $\wedge_{n}$ has global dimension $n$ and that the simple module $S_{1}$ corresponding to the vertex 1 has projective dimension $n$. On one hand, the module $S_{1}$ is a preinjective module (see [2]) and each indecomposable preinjective module has projective dimension $n$. On the other hand, there is a family of indecomposable $\wedge$-modules $\left\{Y_{\lambda} \mid 0 \neq \lambda \in k\right\}$ in $\mathcal{P}^{1}(\wedge)$ of dimension 2 with support containing vertices 1 and 2 only (i.e. the $Y_{\lambda}$ can be considered as modules over double arrows; see [4, sect. 8]). Moreover, it holds that $\operatorname{hom}_{\wedge}\left(Y_{\lambda}, Y_{\mu}\right)=0$ for $\lambda \neq \mu$ and there is a 
nonzero morphism from each $Y_{\lambda}$ to $S_{1}$. Suppose $f: X \rightarrow S_{1}$ is a right $\mathcal{P}^{1}(\wedge)$ approximation. One can easily show that each $Y_{\lambda}$ should appear as a summand of $X$, but this is impossible. Hence $S_{1}$ has no right $\mathcal{P}^{1}(\wedge)$-approximation, that is, $\mathcal{P}^{1}(\wedge)$ is not contravariantly finite in $\bmod \wedge$. Similarly, one can prove that $\mathcal{P}^{i}(\wedge)$ is not contravariantly finite in $\bmod \wedge$ for $2 \leq i \leq n-1$.

An easy observation shows that $\operatorname{pd}_{\wedge} \underline{a}_{i}=n-1$ for $0 \leq i \leq n-2$.

\section{An APplication}

As an application of the sufficient condition given in section 1 , in this section we prove that $\mathcal{P}^{i}(\wedge)$ is contravariantly finite in $\bmod \wedge$ when $\wedge$ is stably equivalent to a hereditary algebra. The proof is based on the following two lemmas.

Lemma 2.1 [1, Proposition 4.12]. For an artin algebra, the following are equivalent.

(a) $\wedge$ satisfies the conditions:

(i) A simple module $S$ is a torsion module (i.e. $\left.S^{*}=\operatorname{hom}_{\wedge}(S, \wedge)=0\right)$ if it is a composition factor of $\underline{r} P / \operatorname{soc} P$ for some indecomposable projective module $P$.

(ii) Every indecomposable torsionless module (i.e. a submodule of a free $\wedge^{-}$ module) is simple or projective.

(b) $\wedge$ is stably equivalent to a hereditary algebra.

Lemma 2.2. Suppose that $\wedge$ is stably equivalent to a hereditary algebra. Then it holds that $\operatorname{pd}_{\wedge} \underline{a}_{i} \leq i$ for each $i \geq 0$, where $\underline{a}_{i}=\tau_{\Omega(\bmod \wedge) \cap \mathcal{P}^{i}(\wedge)}(\underline{r})$.

Proof. By Lemma 2.1 one gets that

$$
\underline{a}_{i}=P \oplus S_{1} \oplus \cdots \oplus S_{t},
$$

where $P$ is projective and the $S_{j}$ are simple modules.

Suppose now that $\operatorname{pd}_{\wedge} \underline{a}_{i}>i$. Then there is a simple module $S:=S_{j}$ satisfying that $\operatorname{pd}_{\wedge} S>i$. By the construction of $\underline{a}_{i}$, there is an epimorphism $f: L \rightarrow S$ with $L$ indecomposable in $\Omega(\bmod \wedge) \cap \mathcal{P}^{i}(\wedge)$. We claim that $f(\operatorname{soc}(L))=0$. Otherwise, $f$ would be a splittable epimorphism, so $\operatorname{pd}_{\wedge} S \leq i$, but this is impossible. Thus $S$ is a composition factor of $L / \operatorname{soc} L$. Since $L$ is in $\Omega(\bmod \wedge)$, it follows that $L \subset \underline{r} Q$ for some projective module $Q$. Then $L / \operatorname{soc} L \subset \underline{r} Q / \operatorname{soc} Q$; that is, $S$ is a composition factor of $\underline{r} Q / \operatorname{soc} Q$. This implies by Lemma 2.1 (ii) that $S$ is a torsion module. This contradicts that $S \subset \underline{a}_{i}$. Therefore, it holds that $\operatorname{pd}_{\wedge} \underline{a}_{i} \leq i$.

Proposition 2.3. Suppose that $\wedge$ is stably equivalent to a hereditary algebra. Then $\mathcal{P}^{i}(\wedge)$ is contravariantly finite in $\bmod \wedge$ for each $i \geq 0$.

Proof. By Lemma 2.2 one knows that $\operatorname{pd}_{\wedge} \underline{a}_{i} \leq i$ for each $i$. Then by applying Proposition 1.3 one gets that $\mathcal{P}^{i+1}(\wedge)$ is contravariantly finite in $\bmod \wedge$. The proposition then follows from the fact that the subcategory $\mathcal{P}^{0}(\wedge)$ is always contravariantly finite in $\bmod \wedge$.

Remark. In [1] it is proved that $\mathcal{P}^{\infty}(\wedge)$ is contravariantly finite in $\bmod \wedge$ when $\wedge$ is stably equivalent to a hereditary algebra. Combining this result with Proposition 2.3 , one can see that if $\wedge$ is stably equivalent to a hereditary algebra, then $\mathcal{P}^{i}(\wedge)$ is contravariantly finite in $\bmod \wedge$ for all $i \in \mathbb{N} \cup\{\infty\}$. 


\section{REFERENCES}

1. M. Auslander and I. Reiten, Applications of contravariantly finite subcategories, Adv. in Math. 86 (1991), 111-152. MR 92e:16009

2. M. Auslander and S. O. Smalø, Preprojective modules over artin algebras, J. Algebra 66 (1980), 61-122. MR 83a:16039

3. — Almost split sequences in subcategories, J. Algebra 69 (1981), 426-454. MR 82j: $16048 \mathrm{a}$

4. P. Gabriel, B. Keller, and A. V. Roiter, Representations of finite-dimensional algebras, Encyclopaedia of Math. Sci., vol. 73, Springer, Berlin, 1992. MR 94h:16001b

5. K. Igusa, S. O. Smalø, and G. Todorov, Finite projectivity and contravariant finiteness, Proc. Amer. Math. Soc. 109 (1990), 937-941. MR 91b:16010

6. S. O. Smalø, Functorial finite subcategories over triangular matrix rings, Proc. Amer. Math. Soc. 111 (1991), 651-656. MR 91f:16016

Department of Mathematics, Beijing Normal University, Beijing 100875, People's Republic of China

E-mail address: dengbm@bnu.ihep.ac.cn 